\title{
A Reed-Solomon Coded DS-CDMA System Using Noncoherent $M$-ary Orthogonal Modulation over Multipath Fading Channels
}

\author{
Lie-Liang Yang, Member, IEEE, Kai Yen, Student Member, IEEE, and Lajos Hanzo, Senior Member, IEEE
}

\begin{abstract}
The performance of Reed-Solomon (RS) coded direct-sequence code division multiple-access (DS-CDMA) systems using noncoherent $M$-ary orthogonal modulation is investigated over multipath Rayleigh fading channels. Diversity reception techniques with equal gain combining (EGC) or selection combining (SC) are invoked and the related performance is evaluated for both uncoded and coded DS-CDMA systems. "Errors-and-erasures" decoding is considered, where the erasures are based on Viterbi's so-called ratio threshold test (RTT). The probability density functions (PDF) of the ratio associated with the RTT conditioned on both the correct detection and erroneous detection of the $M$-ary signals are derived. These PDFs are then used for computing the codeword decoding error probability of the RS coded DS-CDMA system using "errors-and-erasures" decoding. Furthermore, the performance of the "errors-and-erasures" decoding technique employing the RTT is compared to that of "error-correction-only" decoding refraining from using side-information over multipath Rayleigh fading channels. As expected, the numerical results show that when using "errors-and-erasures" decoding, RS codes of a given code rate can achieve a higher coding gain than without erasure information.
\end{abstract}

Index Terms-Direct sequence code division multiple-access, error-correction-only decoding, errors-and-erasures decoding, noncoherent $M$-ary orthogonal signaling, ratio threshold test, Reed-Solomon codes.

\section{INTRODUCTION}

$\mathbf{R}$ ECENTLY, there has been significant interest in the application of code-division multiple-access (CDMA) in cellular and wireless personal communications [1], [2]. CDMA has also been adopted for the third-generation global standard wireless systems. On the uplink of a direct-sequence CDMA (DS-CDMA) cellular system, due to the high complexity of coherent modulation/demodulation which would require a pilot signal for each user, noncoherent $M$-ary orthogonal modulation using $M=64$, i.e., 6-bit symbols, has been proposed (for example) for the reverse link of IS-95 [3]. Analysis of DS-CDMA systems using $M$-ary orthogonal signaling has been provided (for example) in [4]-[10] for both additive white Gaussian noise (AWGN) and multipath fading channels.

In cellular DS-CDMA systems, forward error correction (FEC) is often used for mitigating the effects of fading and

Manuscript received October 25, 1999; revised April 24, 2000. The team's research was supported by EPSRC, U.K.; the Commission of the European Communities, Brussels, Belgium, Motorola ECID, Swindon, U.K.; and the Virtual Centre of Excellence (VCE) in Mobile Communications.

The authors are with the Department of Electronics and Computer Science, University of Southampton, SO17 1BJ, U.K. (e-mail: 1h@ecs.soton.ac.uk).

Publisher Item Identifier S 0733-8716(00)09196-4. interference. The so-called "errors-and-erasures" decoding schemes [11] are often preferable to "error-correction-only" decoding, since typically more erasures than errors can be corrected. Hence, it is beneficial to determine the reliability of the received symbols and to erase the low-reliability symbols prior to the decoding process. There are a number of methods for generating reliability-based side information, and their performance has been analyzed (for example, in [10] and [12]-[20]). An erasure insertion scheme suitable for $M$-ary orthogonal modulation is the ratio-threshold test (RTT), which was proposed by Viterbi [13].

In RTT, an erasure is declared whenever the ratio of the maximum to the second maximum of the inputs of the maximum-likelihood detector (MLD) does not exceed a preset threshold. The RTT was originally proposed in order to mitigate the partial-band interference or multitone interference in $M$-ary frequency shift keying (MFSK) systems, but it was later invoked also in the context of $M$-ary orthogonal signaling in order to generate channel-quality related information. Kim and Stark [10] have employed it also for mitigating the effect of Rayleigh-fading and have analyzed some of the performance limits of an RS coded DS-CDMA system by using "errors-and-erasures" decoding. In [19] the performance of RS codes and redundant residue number system (RRNS) codes was evaluated, when both the RTT and the so-called output threshold test (OTT)-based erasure scheme were considered. It can be shown that the RTT-based erasure scheme's optimum decision threshold is typically in the interval of $[1.5,2]$ for a wide range of signal-to-noise ratio (SNR) per bit, only slightly depending on the SNR per bit value encountered. In this paper, we investigate the performance of an RS coded DS-CDMA system, when $M$-ary orthogonal signaling is employed in conjunction with an RTT-based erasure insertion scheme over multipath Rayleigh-fading channels. Two different diversity combining schemes [8], [21]—namely equal-gain combining (EGC) and selection combining (SC)-are considered, and their performance is evaluated in the context of the proposed RTT based erasure insertion scheme. We derive the probability density function (PDF) of the ratio defined in the RTT conditioned on both the correct detection hypothesis $\left(H_{1}\right)$ and erroneous detection hypothesis $\left(H_{0}\right)$ of the received $M$-ary signals over multipath Rayleigh fading channels. These PDFs are then used to compute the symbol erasure probability and the random symbol error probability after erasure insertion, and finally to compute the codeword decoding error probability. These analytical results allow us to quantify the performance 


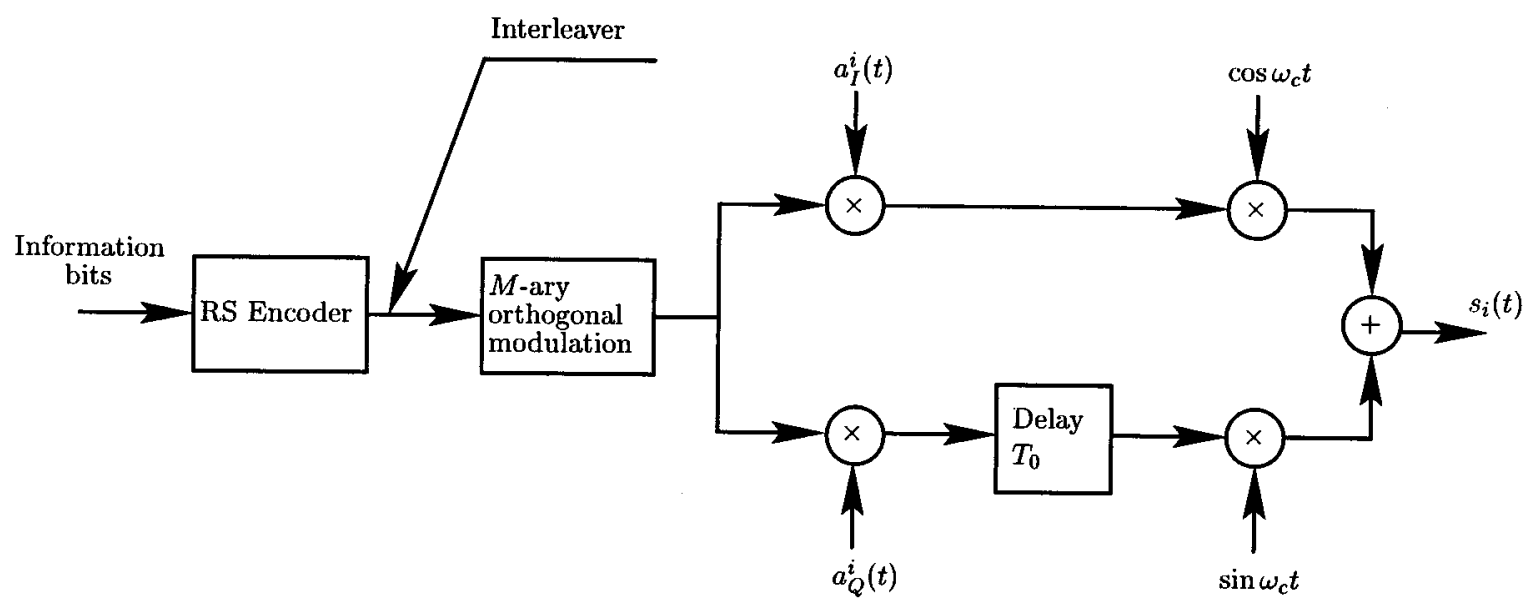

Fig. 1. Transmitter block diagram of the DS-CDMA system using $M$-ary orthogonal modulation, RS encoding, and symbol interleaving.

of the system considered using a numerical approach. Furthermore, with the aid of these analytical results, we gain an insight into the basic characteristics of Viterbi's RTT, and determine the optimum decision threshold for practical systems, since the formulas obtained can be evaluated numerically.

The remainder of this paper is organized as follows. In the next section, the system and channel model are described. Section III addresses the noncoherent detection of DS-CDMA signals and derives the required PDFs associated with EGC and SC based detection. In Section IV, the average error probability of the uncoded system is analyzed, while the expressions of the conditional PDFs of the required ratio associated with the RTT and the RS-codeword decoding error probability are derived in Section V. Numerical results are provided in Section VI, and finally in Section VII we present our conclusions.

\section{SYSTEM DESCRIPTION AND CHANNEL MODEL}

\section{A. The Transmitted Signals}

The transmitter block diagram of the coded $M$-ary orthogonal modulation based DS-CDMA system is shown in Fig. 1. The information bits are first grouped into $b$-bit symbols, where $b=\log _{2} M$. Then, $\mathcal{K}$ information symbols are encoded into an $\mathcal{N}$-symbol RS codeword. In order to randomize the effects of bursty symbol errors, the RS codewords are then interleaved. Finally, each RS coded symbol is $M$-ary modulated, DS spread and carrier-modulated using the approach of [8], in order to form the transmitted signal. Hence, for convenience, we shall adopt notations similar to those used in [8]. In a DS-CDMA system in which there are $K$ active users transmitting their signals simultaneously, the signal transmitted by user $i, i=1,2, \cdots, K$, can be expressed as [8]

$$
\begin{aligned}
s_{i}(t)= & \sqrt{P} W^{j}(t) a_{I}^{i}(t) \cos \omega_{c} t+\sqrt{P} W^{j} \\
& \cdot\left(t-T_{0}\right) a_{Q}^{i}\left(t-T_{0}\right) \sin \omega_{c} t, \quad 0 \leq t \leq T_{s}
\end{aligned}
$$

where

$P \quad$ is the transmitted power,

$T_{s} \quad$ is the symbol duration,

$\omega_{c}$ is the carrier's angular frequency, and

$T_{0} \quad$ is an offset time.
Furthermore, $W^{j}(t)$ is the $j$ th Walsh-Hadamard orthogonal function, which represents the $j$ th orthogonal signal of the $i$ th user's symbols, while $a_{I}^{i}(t)$ and $a_{Q}^{i}(t)$ represent the spreading waveforms of the in-phase $(I)$ and quadrature $(Q)$ phase channels, respectively. These quadrature components are expressed as

$$
\begin{aligned}
& a_{I}^{i}(t)=\sum_{h=-\infty}^{\infty} a_{h}^{I, i} p\left(t-h T_{c}\right) \\
& a_{Q}^{i}(t)=\sum_{h=-\infty}^{\infty} a_{h}^{Q, i} p\left(t-h T_{c}\right)
\end{aligned}
$$

where $a_{h}^{I, i}, a_{h}^{Q, i}$ are assumed to be independent identically distributed (i.i.d.) random variables assuming values of +1 and -1 with equal probability of $1 / 2$. Furthermore, $T_{c}$ represents the chip duration, and $p(t)$ is assumed to be the rectangular chip waveform, which is defined over the interval $\left(0, T_{c}\right]$. Moreover, we assume that $N_{s}=T_{s} / T_{c}=b T / T_{c}=b N$, where $T$ is the bit-duration and $N=T / T_{c}$.

\section{B. The Channel Model}

We assume that the channel between the $i$ th transmitter and the corresponding receiver is a multipath Rayleigh fading channel [22]. The complex lowpass equivalent representation of the impulse response experienced by user $i$ is given by

$$
h_{i}(t)=\sum_{l_{p}=1}^{L_{p}} \alpha_{i l_{p}} \delta\left(t-\tau_{i l_{p}}\right) \exp \left(-j \phi_{i l_{p}}\right)
$$

where $\alpha_{i l_{p}}, \tau_{i l_{p}}$, and $\phi_{i l_{p}}$ represent the attenuation factor, delay and phase-shift for the $l_{p}$ th multipath component of the channel, respectively, while $L_{p}$ is the total number of diversity paths and $\delta(t)$ is the Delta-function. We assume that the $i$ th user's multipath attenuations $\left\{\alpha_{i l_{p}}, l_{p}=1,2, \cdots, L_{p}\right\}$ in (4) are independent Rayleigh-distributed random variables having a PDF given by [22, (14-1-23)]

$$
\begin{aligned}
f\left(\alpha_{i l_{p}}\right) & =M\left(\alpha_{i l_{p}}, \Omega\right) \\
M(R, \Omega) & =\frac{2 R}{\Omega} \exp \left(-\frac{R^{2}}{\Omega}\right)
\end{aligned}
$$




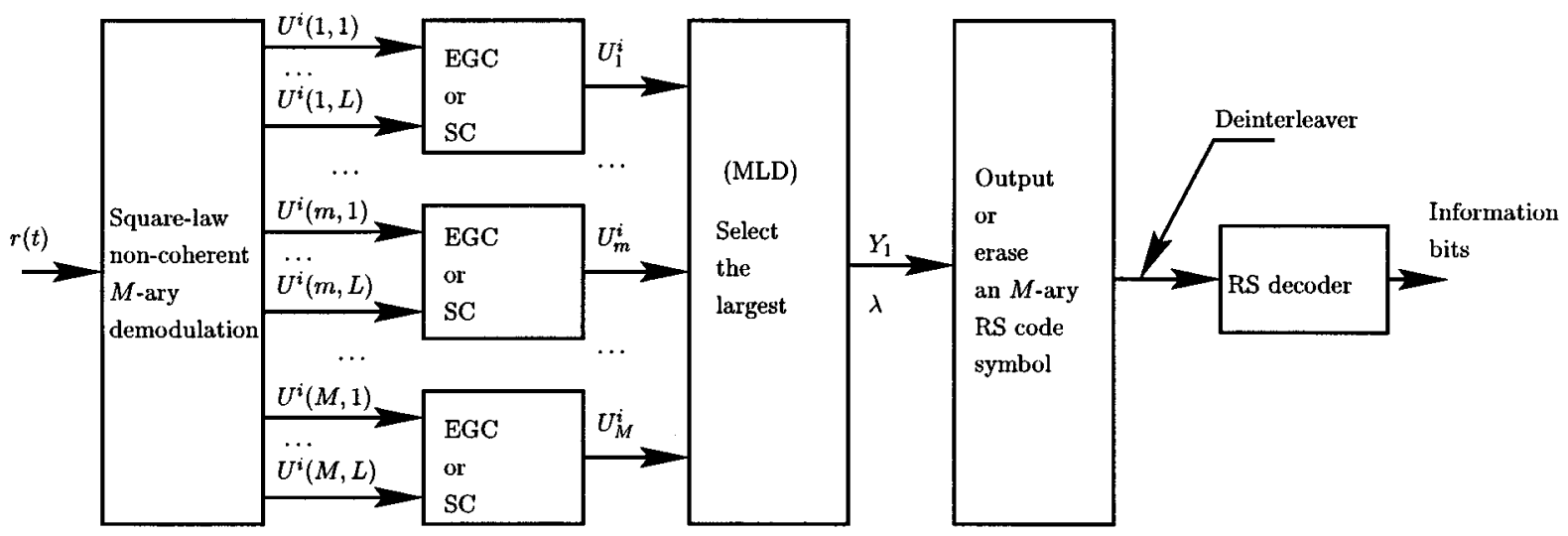

Fig. 2. Receiver model of the DS-CDMA system investigated using noncoherent $M$-ary demodulation, multipath diversity combining, maximum-likelihood detection (MLD), erasure insertion based on the RTT, deinterleaving, and RS "errors-and-erasures" decoding.

where $\Omega=E\left[\left(\alpha_{i l_{p}}\right)^{2}\right]$. The phases $\left\{\phi_{i l_{p}}, l_{p}=1,2, \cdots, L_{p}\right\}$ of the different paths are assumed to be uniformly distributed random variables in $[0,2 \pi)$, while the $i$ th user's path delays of $\left\{\tau_{i l_{p}}, l_{p}=1,2, \cdots, L_{p}\right\}$ are modeled as random variables that are mutually independent of each other and uniformly distributed in $\left[0, T_{s}\right)$. We also assume that ideal power control is employed, in order that the received signal powers are the same for all $K$ users. Then the received signal at the base station generated by the $K$ users can be expressed as

$$
r(t)=\sum_{i=1}^{K} y_{i}(t)+n(t)
$$

where

$$
\begin{aligned}
y_{i}(t)= & \sum_{l_{p}=1}^{L_{p}} \sqrt{P} \alpha_{i l_{p}}\left[W^{j}\left(t-\tau_{i l_{p}}\right) a_{I}^{i}\left(t-\tau_{i l_{p}}\right)\right. \\
& \cdot \cos \left(\omega_{c}\left(t-\tau_{i l_{p}}\right)-\phi_{i l_{p}}\right)+W^{j}\left(t-T_{0}-\tau_{i l_{p}}\right) \\
& \left.\cdot a_{Q}^{i}\left(t-T_{0}-\tau_{i l_{p}}\right) \sin \left(\omega_{c}\left(t-\tau_{i l_{p}}\right)-\phi_{i l_{p}}\right)\right]
\end{aligned}
$$

$n(t)$ represents the AWGN, which is modeled as a random variable with zero mean and double-sided power spectral density of $N_{0} / 2$. After the receiver's bandpass filter, the noise $n(t)$ becomes a narrow-band noise process, which can be expressed as [8]

$$
n(t)=n_{c}(t) \cos \omega_{c} t+n_{s}(t) \sin \omega_{c} t
$$

where $n_{c}(t)$ and $n_{s}(t)$ represent low-pass-filtered Gaussian processes.

\section{The Detection Model}

The receiver schematic of the studied DS-CDMA system including noncoherent $M$-ary demodulation, multipath diversity combining, maximum-likelihood detection (MLD), RTT-based erasure insertion, deinterleaving, and RS "errors-and-erasures" decoding is shown in Fig. 2, where the square-law-based noncoherent $M$-ary demodulation block is the same as that used in [8], and hence the interested readers are referred to [8] for further details. Multipath diversity combining invoking the EGC or the SC principle was implemented in the "EGC or SC" block. The MLD block selects the largest from its input variables and computes the value of $\lambda$-the ratio of the largest to the second largest of the MLDs inputs. Following the MLD stage, the next block may output an $M$-ary RS code symbol or insert an erasure. Finally, after symbol-based deinterleaving, the RS decoder invokes "errors-and-erasures" decoding and then outputs the received information bits.

As shown in [8, Fig. 4], the outputs of the low-pass filters of the in-phase $(I)$ and quadrature $(Q)$ channels are first multiplied by the spreading sequences, and the resulting signals are correlated with each of the $M$ orthogonal Walsh-Hadamard functions. Let the $i$ th user be the user of interest and assume that the $j$ th symbol was transmitted. According to Jalloul and Holtzman [8], the output variable of the $l$ th branch of the $m$ th correlator in Fig. 2 can be expressed as [8]

$$
\begin{aligned}
U^{i}(m, l)= & {\left[\int_{\tau_{i l}}^{T_{s}+\tau_{i l}} r(t) a_{I}^{i} W^{m} \cos \omega_{c} t d t\right.} \\
& \left.+\int_{\tau_{i l}}^{T_{s}+\tau_{i l}} r(t) a_{Q}^{i} W^{m} \sin \omega_{c} t d t\right]^{2} \\
& +\left[\int_{\tau_{i l}}^{T_{s}+\tau_{i l}} r(t) a_{Q}^{i} W^{m} \cos \omega_{c} t d t\right. \\
& \left.\quad-\int_{\tau_{i l}}^{T_{s}+\tau_{i l}} r(t) a_{I}^{i} W^{m} \sin \omega_{c} t d t\right]^{2} \\
& \quad m=1,2, \cdots, M ; l=1,2, \cdots, L .
\end{aligned}
$$

Upon assuming that Gaussian approximation of the multipath interference and that of the multiple access interference can be employed, consequently, $U^{i}(m, l)$ is the sum of the square of two Gaussian random variables, each having a variance of [8] [(28)]w

$$
\sigma^{2}=\frac{N_{0}}{2}+\frac{E_{s} \Omega}{3 N_{s}}\left(K L_{p}-1\right)
$$

where $\Omega=E\left[\left(\alpha_{i l_{p}}\right)^{2}\right]$, as we assumed in (5).

Since $U^{i}(m, l)$ is the sum of the square of two Gaussian random variables, it can be shown that, conditioned on $\alpha_{i l}$, $U^{i}(m, l)$ becomes Chi-square distributed [22] with two degrees of freedom, and its PDF can be expressed as

$$
\begin{aligned}
& f_{U^{i}(m, l)}\left(y \mid \mathcal{R}_{i l}\right) \\
& \quad=\frac{1}{2 \sigma^{2}} \exp \left(-\frac{y+\mathcal{R}_{i l}}{2 \sigma^{2}}\right) I_{0}\left(\frac{\sqrt{y \mathcal{R}_{i l}}}{\sigma^{2}}\right), \quad y \geq 0
\end{aligned}
$$


if $m=j$, where $\mathcal{R}_{i l}=E_{s} \alpha_{i l}^{2}$ is the noncentrality parameter of the Chi-square distribution and $I_{0}(\cdot)$ is the modified Bessel function of the zeroth order. If $m \neq j$, then [22]

$$
f_{U^{i}(m, l)}(y)=\frac{1}{2 \sigma^{2}} \exp \left(-\frac{y}{2 \sigma^{2}}\right), \quad y \geq 0 .
$$

Using $2 \sigma^{2}$ to normalize $U^{i}(m, l)$, we obtain the normalized PDFs of $U^{i}(m, l)$ as

$$
\begin{aligned}
& f_{U^{i}(m, l)}\left(y \mid \gamma_{i l}\right)= \exp \left(-\left[y+\gamma_{i l}\right]\right) I_{0}\left(\sqrt{4 y \gamma_{i l}}\right), \\
& m=j, y \geq 0 \\
& f_{U^{i}(m, l)}(y)=\exp (-y), \quad m \neq j, y \geq 0
\end{aligned}
$$

where

$$
\gamma_{i l}=\frac{\mathcal{R}_{i l}}{2 \sigma^{2}}=\bar{\gamma}_{c} \cdot \frac{\alpha_{i l}^{2}}{\Omega}
$$

and

$$
\bar{\gamma}_{c}=\left[\frac{2}{3 N_{s}}\left(K L_{p}-1\right)+\left(\frac{E_{s} \Omega}{N_{0}}\right)^{-1}\right]^{-1} .
$$

Since $\left\{\alpha_{i l}\right\}$ are independent Rayleigh-distributed random variables, $\left\{\alpha_{i l}^{2}\right\}$ are exponentially distributed random variables. Consequently, with the aid of (5), it can be shown that the PDF of $\gamma_{i l}$ defined in (15) can be expressed as

$$
f\left(\gamma_{i l}\right)=\frac{1}{\bar{\gamma}_{c}} \exp \left(-\frac{\gamma_{i l}}{\bar{\gamma}_{c}}\right)
$$

\section{A. Equal Gain Combining}

For a receiver using $L$ th-order $\left(L \leq L_{p}\right)$ diversity reception and EGC, the $L$ branches are equally weighted and then added in order to form the decision variables, which can be expressed as

$$
U_{m}^{i}=\sum_{l=1}^{L} U^{i}(m, l)
$$

for $m=1,2, \cdots, M$. Assuming that the $j$ th symbol was transmitted, then, since the quantities $\left\{U^{i}(m, l)\right\}$ are Chi-square distributed random variables with two degrees of freedom and have a PDF given by (13) and (14) for $m=j$ and $m \neq j$, respectively, it can be shown that, for a given set of multipath attenuations $\left\{\alpha_{i 1}, \alpha_{i 2}, \cdots, \alpha_{i L}\right\}, U_{m}^{i}$ is Chi-square distributed with $2 L$ degrees of freedom, and it obeys the normalized PDF of [22]

$$
\begin{aligned}
f_{U^{i}}(y \mid \gamma) & =\left(\frac{y}{\gamma}\right)^{(L-1) / 2} \exp (-[y+\gamma]) I_{L-1}(\sqrt{4 y \gamma}) \\
y \geq 0 & \\
f_{U_{m}^{i}}(y) & =\frac{1}{(L-1) !} y^{L-1} \exp (-y) \\
& \neq j, y \geq 0
\end{aligned}
$$

where $I_{L}(\cdot)$ is the modified Bessel function of the $L$ th order [22], and

$$
\gamma=\sum_{l=1}^{L} \gamma_{i l}
$$

where the PDF of $\gamma_{i l}$ is given by (17). We assume throughout that all the path gains are i.i.d. random variables, which in turn means that $\left\{\gamma_{i l}\right\}$ are i.i.d. random variables. Consequently, it can be readily shown that $\gamma$ obeys the central Chi-square distribution with $2 L$ degrees of freedom [22], which can be expressed as

$$
f(\gamma)=\frac{1}{(L-1) ! \bar{\gamma}_{c}^{L}} \gamma^{L-1} \exp \left(-\frac{\gamma}{\bar{\gamma}_{c}}\right)
$$

Since the decision variables $\left\{U_{1}^{i}, U_{2}^{i}, \cdots, U_{M}^{i}\right\}$ are independent random variables, the conditioning in (19) may be removed ${ }^{1}$ by averaging $f_{U_{j}^{i}}(y \mid \gamma)$ over the valid range of $\gamma$, which can be expressed as

$$
f_{U_{j}^{i}}(y)=\int_{0}^{\infty} f_{U_{j}^{i}}(y \mid \gamma) f(\gamma) d \gamma
$$

Upon substituting (19) and (22) into (23), it can be shown that

$$
\begin{gathered}
f_{U_{j}^{i}}(y)=\frac{1}{(L-1) !\left(1+\bar{\gamma}_{c}\right)^{L}} y^{L-1} \exp \left(-\frac{y}{1+\bar{\gamma}_{c}}\right), \\
y \geq 0 .
\end{gathered}
$$

\section{B. Selection Combining}

For an $L$-branch diversity receiver with $\mathrm{SC}$-where the branch signal having the largest amplitude is selected for demodulation - the conventional decision variable of an $M$-ary orthogonal modulation system is defined as

$$
\begin{array}{r}
U^{i}=\max \left\{U^{i}(1,1), U^{i}(1,2), \cdots, U^{i}(1, L), \ldots,\right. \\
\left.U^{i}(M, 1), U^{i}(M, 2), \cdots, U^{i}(M, L)\right\} .
\end{array}
$$

However, this definition is not suitable for generating reliability information by invoking Viterbi's RTT, since there exist $L$ branch signals, which are matched to the transmitted signal. This issue will become explicit during our further discussions. Hence, in this paper, a two-stage maximum selection scheme is proposed, in order to invoke the RTT. The first-stage maximum selection is defined as the maximum selection from the $L$ diversity components for each specific given $m$, i.e.,

$$
U_{m}^{i}=\max \left\{U^{i}(m, 1), U^{i}(m, 2), \ldots, U^{i}(m, L)\right\}
$$

for $m=1,2, \cdots, M$. Explicitly, after the above selection process, only one output, namely $U_{j}^{i}$ is matched to the transmitted signal and the other $(M-1)$ outputs are mismatched with respect to the transmitted signal, and hence the RTT [13] can be invoked, in order to obtain the required reliability measures. Based on the above maximum selection, the symbol is decided correctly, if and only if the output $U_{j}^{i}$, which is matched to the transmitted signal-remembering that the $j$ th symbol was

${ }^{1}$ Note that since we have assumed that $\left\{\alpha_{i l_{p}}\right\}$ are independent random variables and we also assumed that $\left\{U_{1}^{i}, U_{2}^{i}, \ldots, U_{M}^{i}\right\}$ are independent random variables, the conditioning due to the fading of the channel can be removed at the branch outputs by averaging the PDF of $U^{i}(m, l)$ for $m=j$ in (13) over the valid range of $\gamma_{i l}$, which results in $f_{U^{i}(m, l)}(y)=\left(1 / 1+\bar{\gamma}_{c}\right) \exp (-(y / 1+$ $\left.\bar{\gamma}_{c}\right)$ ) for $m=j$. The approach of this section can be followed in order to derive (24) and (31). 
transmitted-is larger than any other outputs mismatched to the transmitted symbol. Hence, the second-stage maximum selection finds the largest one from the set $\left\{U_{1}^{i}, U_{2}^{i}, \ldots, U_{M}^{i}\right\}$, which can be expressed as

$$
U^{i}=\max \left\{U_{1}^{i}, U_{2}^{i}, \ldots, U_{M}^{i}\right\}
$$

Let us now derive the PDFs of $U_{m}^{i}$. Since the normalized PDF of $U^{i}(m, l)$ for $l=1,2, \ldots, L$ was given by (14), the PDF of $U_{m}^{i}$ for $m \neq j$ can be expressed as

$$
\begin{aligned}
f_{U_{m}^{i}}(y) & =\frac{d}{d y}[1-\exp (-y)]^{L} \\
& =L \exp (-y)[1-\exp (-y)]^{L-1} .
\end{aligned}
$$

Similarly, the conditional PDF of $U_{j}^{i}$ can be derived, which can be expressed as

$$
\begin{aligned}
f_{U_{j}^{i}}\left(y \mid \gamma_{i 1}, \gamma_{i 2}, \ldots, \gamma_{i L}\right) & =\frac{d}{d y}\left[\prod_{l=1}^{L} \int_{0}^{y} f_{U^{i}(j, l)}\left(x \mid \gamma_{i l}\right) d x\right] \\
= & \sum_{l=1}^{L} f_{U^{i}(j, l)}\left(y \mid \gamma_{i l}\right)\left[\prod_{\substack{u=1 \\
u \neq l}}^{L} \int_{0}^{y} f_{U^{i}(j, u)}\left(x \mid \gamma_{i u}\right) d x\right]
\end{aligned}
$$

where $f_{U^{i}(j, u)}\left(x \mid \gamma_{i u}\right)$ for $u=1,2, \ldots, L$ was given by (13). The conditioning with respect to $\left\{\gamma_{i l}\right\}$ can be removed ${ }^{2}$ at this stage by integrating $f_{U_{i}^{i}}\left(y \mid \gamma_{i 1}, \gamma_{i 2}, \ldots, \gamma_{i L}\right)$ over the valid ranges of $\gamma_{i 1}, \gamma_{i 2}, \ldots, \gamma_{i L}$, which can be expressed as

$$
\begin{aligned}
f_{U_{j}^{i}}(y)= & \underbrace{\int \cdots \int_{0}^{\infty} \sum_{l=1}^{L} f_{U^{i}(j, l)}\left(y \mid \gamma_{i l}\right)}_{L} \\
& \cdot\left[\prod_{\substack{u=1 \\
u \neq l}}^{L} \int_{0}^{y} f_{U^{i}(j, u)}\left(x \mid \gamma_{i u}\right) d x\right] \\
& \cdot f\left(\gamma_{i 1}\right) f\left(\gamma_{i 2}\right) \cdots f\left(\gamma_{i L}\right) d \gamma_{i 1} d \gamma_{i 2} \cdots d \gamma_{i L} .
\end{aligned}
$$

Substituting $f_{U^{i}(j, l)}\left(x \mid \gamma_{i l}\right)$ and $f\left(\gamma_{i l}\right)$ from (13) and (17) into (30), and remembering that $\left\{\gamma_{i l}\right\}$ are i.i.d. random variables, (30) can be simplified to

$$
\begin{aligned}
f_{U_{j}^{i}}(y)= & \frac{L}{1+\bar{\gamma}_{c}} \exp \left(-\frac{y}{1+\bar{\gamma}_{c}}\right) \\
& \cdot\left[1-\exp \left(-\frac{y}{1+\bar{\gamma}_{c}}\right)\right]^{L-1}, \quad y \geq 0
\end{aligned}
$$

where $\bar{\gamma}_{c}$ is given by (16).

Above we have derived the PDFs of the decision variables that are input to the MLD block. With the aid of these PDFs, the average symbol error probabilities can now be derived for both EGC and SC.

\footnotetext{
${ }^{2}$ See footnote 1 .
}

\section{Average Error Probability Without FEC}

Let $\left\{U_{1}^{i}, U_{2}^{i}, \cdots, U_{M}^{i}\right\}$ represent the decision variables after diversity combining, which are input to the MLD block, and assume that the $j$ th symbol is transmitted. Let $H_{1}$ and $H_{0}$ represent the hypotheses of correct decision and erroneous decision of the MLD block. Then the average correct symbol probability of $P\left(H_{1}\right)$ and erroneous symbol probability of $P\left(H_{0}\right)$ can be expressed as

$$
\begin{gathered}
P\left(H_{1}\right)=P\left(U_{1}^{i}<U_{j}^{i}, \ldots, U_{j-1}^{i}<U_{j}^{i},\right. \\
\left.U_{j+1}^{i}<U_{j}^{i}, \ldots, U_{M}^{i}<U_{j}^{i}\right) \\
P\left(H_{0}\right)=1-P\left(H_{1}\right)
\end{gathered}
$$

respectively. Given the symbol error probability, the average bit error rate (BER) can be expressed as [22]

$$
P_{b}=\frac{2^{b-1}}{M-1} P\left(H_{0}\right)
$$

\section{A. Equal Gain Combining}

For EGC, referring to (32), the probability of $P\left(H_{1}\right)$ can be expressed as

$$
P\left(H_{1}\right)=\int_{0}^{\infty} f_{U_{j}^{i}}(y)\left[\int_{0}^{y} f_{U_{m}^{i}}(x) d x\right]^{M-1} d y
$$

where $f_{U_{m}^{i}}(x)$ and $f_{U_{j}^{i}}(y)$ are given by (20) and (24), respectively. Substituting them into the above equation, it can be shown that

$$
\begin{aligned}
P\left(H_{1}\right)= & \int_{0}^{\infty} \frac{1}{\left(1+\bar{\gamma}_{c}\right)^{L}(L-1) !} y^{L-1} \exp \left(-\frac{y}{1+\bar{\gamma}_{c}}\right) \\
& \cdot\left[1-\exp (-y) \sum_{k=0}^{L-1} \frac{y^{k}}{k !}\right]^{M-1} d y .
\end{aligned}
$$

The average symbol error probability and bit error probability can be computed using (33) and (34), respectively.

\section{B. Selection Combining}

For SC, referring to (32), the probability of $P\left(H_{1}\right)$ can also be expressed in the form of (35), where $f_{U_{m}^{i}}(x)$ and $f_{U_{j}^{i}}(y)$ are given by (28) and (31), respectively. Upon substituting them into (35), the correct symbol probability can be simplified to

$$
P\left(H_{1}\right)=\sum_{m=1}^{L}(-1)^{m+1}\left(\begin{array}{c}
L \\
m
\end{array}\right) \prod_{n=1}^{L(M-1)}\left(\frac{n}{n+m /\left(1+\bar{\gamma}_{c}\right)}\right) .
$$

The derivation of (37) is provided in the Appendix. ${ }^{3}$

The average symbol error probability and bit error probability can be computed with the aid of (33) and (34).

So far, we have obtained the expression of the average error probability per bit, while deriving some further expressions for

\footnotetext{
${ }^{3}$ The erroneous symbol probability, $P\left(H_{0}\right)$, of the $\mathrm{SC}$ scheme has been given in $[23,(11)]$ without detailed derivation.
} 
our forthcoming study of the coded DS-CDMA system using $M$-ary orthogonal modulation. Hence, let us now analyze the performance of the system in conjunction with RS coding using both "error-correction-only" decoding and "errors-and-erasures" decoding by invoking Viterbi’s RTT techniques [13].

\section{PERFORMANCE USING RS FORWARD ERROR-CORRECTION CODES}

Reed-Solomon codes [11] constitute an efficient class of linear codes using multibit symbols and having the capability of correcting/detecting symbol errors and symbol erasures. An $\operatorname{RS}(\mathcal{N}, \mathcal{K})$ code-where $\mathcal{N}$ is the total length of the codeword and $\mathcal{K}$ is the number of information symbols, respectively-can correct up to $t_{c}=\lfloor(\mathcal{N}-\mathcal{K}) / 2\rfloor$ random symbol errors, where $\lfloor x\rfloor$ represents the largest integer not exceeding $x$, or detect up to $(\mathcal{N}-\mathcal{K})$ symbol errors. Alternatively, it is capable of correcting up to $(\mathcal{N}-\mathcal{K})$ symbol erasures. Moreover, it is capable of correcting $t$ or less random symbol errors and $e$ symbol erasures, simultaneously, provided that $2 t+e \leq(\mathcal{N}-\mathcal{K})$. Typically, "errors-and-erasures" decoding is preferable to "error-correction-only" decoding, since more erasures than errors can be corrected. Hence, it is advantageous to determine the reliability of the received $\mathrm{RS}$-coded symbols and to erase the low-reliability symbols prior to the decoding process. Consequently, in this section, both "error-correction-only" decoding and "errors-and-erasures" decoding based on the RTT will be discussed, while their performance comparison will be given in Section VI. Let us first analyze the decoding performance of RS codes using "error-correction-only" decoding.

\section{A. Error-Correction-Only Decoding}

Assuming that sufficiently long channel interleaving was invoked, in order to result in independent symbol errors in an RS codeword, the codeword decoding error probability after "error-correction-only" decoding can be expressed using [22, (8-1-119)] as follows:

$$
P_{W}=\sum_{n=\lfloor(\mathcal{N}-\mathcal{K}) / 2\rfloor+1}^{\mathcal{N}}\left(\begin{array}{l}
\mathcal{N} \\
n
\end{array}\right)\left[P\left(H_{0}\right)\right]^{n}\left[1-P\left(H_{0}\right)\right]^{\mathcal{N}-n}
$$

where the random symbol error probability $P\left(H_{0}\right)$ before decoding is given by (33).

\section{B. Errors-and-Erasures Decoding}

In order to take advantage of the powerful "errors-and-erasures" correction capability of the RS code concerned, it is essential to design an efficient erasure insertion scheme. In this subsection, erasure insertion using Viterbi's RTT [13] is investigated, and the PDFs of the quantities involved in Viterbi's RTT are derived.

Let $\left\{U_{1}^{i}, U_{2}^{i}, \ldots, U_{M}^{i}\right\}$ represent the decision variables input to the MLD block of Fig. 2. The ratio involved in Viterbi's RTT is computed according to the following definition [13]:

$$
\lambda=\frac{Y_{1}=\max _{1}\left\{U_{1}^{i}, U_{2}^{i}, \ldots, U_{M}^{i}\right\}}{Y_{2}=\max _{2}\left\{U_{1}^{i}, U_{2}^{i}, \ldots, U_{M}^{i}\right\}}
$$

where $Y_{1}=\max _{1}\{\cdot\}$ and $Y_{2}=\max _{2}\{\cdot\}$ represent the maximum and the "second" maximum of the decision variables of
$\left\{U_{1}^{i}, U_{2}^{i}, \ldots, U_{M}^{i}\right\}$, respectively. Viterbi [13] pointed out that the demodulated symbols having relatively high ratio of $\lambda$ were more reliable than those having relatively low values of $\lambda$. Consequently, a preset threshold $\lambda_{T}$ can be invoked, in order to erase these low-reliability symbols associated with a ratio of $\lambda \leq \lambda_{T}$, which constitutes the so-called RTT. In this subsection we derive the PDFs of $\lambda$ under the hypotheses $H_{1}$ of correct decision and $H_{0}$ of erroneous decision, and under the assumption that the decision variables are independent random variables [19]. With the aid of these PDFs, not only can the characteristics of the threshold $\lambda_{T}$ be investigated, but also the RS decoding performance can be quantified, while determining the optimum thresholds using a numerical approach.

The PDFs of the maximum and the "second maximum" of $Y_{1}=\max _{1}\{\cdot\}$ and $Y_{2}=\max _{2}\{\cdot\}$ under the hypotheses $H_{1}$ of correct decision and $H_{0}$ of erroneous decision have been given in [19, Appendix] for EGC. For SC, the corresponding PDFs can be derived following a similar approach to that of EGC. Hence, here we simply summarize the corresponding results.

$E G C$ : The PDFs of $Y_{1}$ and $Y_{2}$ conditioned on the hypothesis of $H_{1}$ are given by

$$
\begin{aligned}
& f_{Y_{1}}\left(y \mid H_{1}\right)= \frac{1}{P\left(H_{1}\right)} \cdot \frac{1}{\left(1+\bar{\gamma}_{c}\right)^{L}(L-1) !} y^{L-1} \\
& \cdot \exp \left(-\frac{y}{1+\bar{\gamma}_{c}}\right)[1-\Psi(y)]^{M-1}, \\
& f_{Y_{2}}\left(y \mid H_{1}\right)= \frac{1}{P\left(H_{1}\right)} \cdot \frac{M-1}{(L-1) !} y^{L-1} \exp (-y) \\
& \cdot[1-\Psi(y)]^{M-2} \Psi\left(\frac{y}{1+\bar{\gamma}_{c}}\right), \\
& y \geq 0
\end{aligned}
$$

where $P\left(H_{1}\right)$ is the a priori probability of the hypothesis $H_{1}$ for EGC, which is given by (36), while the short-hand $\Psi(x)$ is defined as

$$
\Psi(x)=\exp (-x) \sum_{k=0}^{L-1} \frac{x^{k}}{k !} .
$$

EGC: The PDFs of $Y_{1}$ and $Y_{2}$ conditioned on the hypothesis of $H_{0}$ are as follows:

$$
\begin{aligned}
f_{Y_{1}}\left(y \mid H_{0}\right) & \frac{1}{P\left(H_{0}\right)} \cdot \frac{M-1}{(L-1) !} y^{L-1} \exp (-y)[1-\Psi(y)]^{M-2} \\
& \cdot\left[1-\Psi\left(\frac{y}{1+\bar{\gamma}_{c}}\right)\right], \quad y \geq 0 \\
f_{Y_{2}}\left(y \mid H_{0}\right) & \frac{1}{P\left(H_{0}\right)} \cdot \frac{M-1}{(L-1) !} y^{L-1} \Psi(y)[1-\Psi(y)]^{M-3} \\
& \cdot\left\{\frac{1}{\left(1+\bar{\gamma}_{c}\right)^{L}} \exp \left(-\frac{y}{1+\bar{\gamma}_{c}}\right)[1-\Psi(y)]+(M-2)\right. \\
& \left.\cdot \exp (-y)\left[1-\Psi\left(\frac{y}{1+\bar{\gamma}_{c}}\right)\right]\right\}, \quad y \geq 0
\end{aligned}
$$

where $P\left(H_{0}\right)$ is the a priori probability of the hypothesis $H_{0}$ for EGC, which is given by (33) with $P\left(H_{1}\right)$ described by (36). 
$S C$ : The PDFs of $Y_{1}$ and $Y_{2}$ conditioned on the hypothesis of $H_{1}$ are formulated as

$$
\begin{aligned}
f_{Y_{1}}\left(y \mid H_{1}\right) & \\
= & \frac{1}{P\left(H_{1}\right)} \cdot \frac{L}{1+\bar{\gamma}_{c}} \exp \left(-\frac{y}{1+\bar{\gamma}_{c}}\right) \\
& \cdot\left[1-\exp \left(-\frac{y}{1+\bar{\gamma}_{c}}\right)\right]^{L-1}[1-\Omega(y)]^{M-1} \quad y \geq 0
\end{aligned}
$$

$$
\begin{aligned}
& f_{Y_{2}}\left(y \mid H_{1}\right) \\
& =\frac{1}{P\left(H_{1}\right)} \cdot L(M-1) \exp (-y)[1-\exp (-y)]^{L-1} \\
& \quad \Omega\left(\frac{y}{1+\bar{\gamma}_{c}}\right)[1-\Omega(y)]^{M-2}, \quad y \geq 0
\end{aligned}
$$

where $P\left(H_{1}\right)$ is the a priori probability of the hypothesis of $H_{1}$ for SC, which is given by (37), and the short-hand $\Omega(x)$ was defined as

$$
\begin{aligned}
\Omega(x) & =\sum_{k=1}^{L}(-1)^{k+1}\left(\begin{array}{l}
L \\
k
\end{array}\right) \exp (-k x) \\
& =1-[1-\exp (-x)]^{L} .
\end{aligned}
$$

$S C$ : The PDFs of $Y_{1}$ and $Y_{2}$ conditioned on the hypothesis of $H_{0}$ can be shown to obey

$$
\begin{aligned}
f_{Y_{1}}\left(y \mid H_{0}\right) & \frac{1}{P\left(H_{0}\right)} \cdot L(M-1) \exp (-y)[1-\exp (-y)]^{L-1} \\
\cdot & {\left[1-\Omega\left(\frac{y}{1+\bar{\gamma}_{c}}\right)\right][1-\Omega(y)]^{M-2}, \quad y \geq 0 } \\
f_{Y_{2}}\left(y \mid H_{0}\right) & \\
= & \frac{1}{P\left(H_{0}\right)} \cdot L(M-1) \Omega(y)[1-\Omega(y)]^{M-3} \\
\cdot & \left\{\frac{1}{1+\bar{\gamma}_{c}} \exp \left(-\frac{y}{1+\bar{\gamma}_{c}}\right)\left[1-\exp \left(-\frac{y}{1+\bar{\gamma}_{c}}\right)\right]^{L-1}\right. \\
& \cdot[1-\Omega(y)]+(M-2) \exp (-x)[1-\exp (-x)]^{L-1} \\
& \left.\cdot\left[1-\Omega\left(\frac{y}{1+\bar{\gamma}_{c}}\right)\right]\right\}, \quad y \geq 0
\end{aligned}
$$

where $P\left(H_{0}\right)$ is the a priori probability of the hypothesis $H_{0}$ for SC, which is given by (33) with $P\left(H_{1}\right)$ formulated in (37).

The PDF of $\lambda$ defined in (39) can now be derived by using the following equation:

$$
\begin{aligned}
f_{\lambda}\left(y \mid H_{\vartheta}\right)= & \frac{1}{P\left(Y_{2}<Y_{1} \mid H_{\vartheta}\right)} \int_{0}^{\infty} y_{2} f_{Y_{1}} \\
& \cdot\left(y_{2} y \mid H_{\vartheta}\right) f_{Y_{2}}\left(y_{2} \mid H_{\vartheta}\right) d y_{2}, \quad y \geq 1
\end{aligned}
$$

as shown in [19, Appendix] for EGC scheme, where $H_{\vartheta} \in$ $\left\{H_{1}, H_{0}\right\}, P\left(Y_{2}<Y_{1} \mid H_{\vartheta}\right)$ represents the probability of $Y_{2}<$ $Y_{1}$ conditioned on the hypothesis of $H_{\vartheta}$. Substituting the corre- sponding PDFs into (50), the PDFs of $\lambda$ under the hypotheses of $H_{1}$ and $H_{0}$ can be expressed as

$$
\begin{aligned}
& f_{\lambda}\left(y \mid H_{1}\right)=C_{H_{1}} \times g_{\lambda}\left(y \mid H_{1}\right), \quad y \geq 1 \\
& f_{\lambda}\left(y \mid H_{0}\right)=C_{H_{0}} \times g_{\lambda}\left(y \mid H_{0}\right), \quad y \geq 1
\end{aligned}
$$

where $g_{\lambda}\left(y \mid H_{1}\right)$ and $g_{\lambda}\left(y \mid H_{0}\right)$ can be shown to obey the following.

$E G C$ :

$$
\begin{aligned}
g_{\lambda}\left(y \mid H_{1}\right)= & y^{L-1} \int_{0}^{\infty} x^{2 L-1} \exp \left(-x-\frac{x y}{1+\bar{\gamma}_{c}}\right) \\
& \cdot[1-\Psi(x y)]^{M-1}[1-\Psi(x)]^{M-2} \Psi\left(\frac{x}{1+\bar{\gamma}_{c}}\right) d x \\
g_{\lambda}\left(y \mid H_{0}\right)= & y^{L-1} \int_{0}^{\infty} x^{2 L-1} \exp (-x y)[1-\Psi(x y)]^{M-2} \\
& {\left[1-\Psi\left(\frac{x y}{1+\bar{\gamma}_{c}}\right)\right] \Psi(x)[1-\Psi(x)]^{M-3} } \\
& \left\{\frac{1}{\left(1+\bar{\gamma}_{c}\right)^{L}} \exp \left(-\frac{x}{1+\bar{\gamma}_{c}}\right)[1-\Psi(x)]\right. \\
& \left.+(M-2) \exp (-x)\left[1-\Psi\left(\frac{x}{1+\bar{\gamma}_{c}}\right)\right]\right\} d x .
\end{aligned}
$$

$S C$ :

$$
\begin{aligned}
g_{\lambda}\left(y \mid H_{1}\right) & \int_{0}^{\infty}\left[\left(1-\exp \left(-\frac{x y}{1+\bar{\gamma}_{c}}\right)\right)(1-\exp (-x))\right]^{L-1} \\
& \cdot[1-\Omega(x y)]^{M-1} \Omega\left(\frac{x}{1+\bar{\gamma}_{c}}\right)[1-\Omega(x)]^{M-2} x \\
& \cdot \exp \left(-x-\frac{x y}{1+\bar{\gamma}_{c}}\right) d x \\
g_{\lambda}\left(y \mid H_{0}\right) & \int_{0}^{\infty}[1-\exp (-x y)]^{L-1}\left[1-\Omega\left(\frac{x y}{1+\bar{\gamma}_{c}}\right)\right] \\
& \cdot[1-\Omega(x y)]^{M-2} \Omega(x) \cdot[1-\Omega(x)]^{M-3} \\
& \cdot\left\{\frac{1}{1+\bar{\gamma}_{c}} \exp \left(-\frac{x}{1+\bar{\gamma}_{c}}\right)\left[1-\exp \left(-\frac{x}{1+\bar{\gamma}_{c}}\right)\right]^{L-1}\right. \\
& \cdot[1-\Omega(x)]+(M-2) \exp (-x)[1-\exp (-x)]^{L-1} \\
& \left.\cdot\left[1-\Omega\left(\frac{x}{1+\bar{\gamma}_{c}}\right)\right]\right\} x \exp (-x y) d x .
\end{aligned}
$$

Upon integrating both sides of (51) and (52) from one to infinity, we obtain:

$$
\begin{aligned}
C_{H_{1}} & =\frac{1}{\int_{1}^{\infty} g_{\lambda}\left(y \mid H_{1}\right) d y} \\
C_{H_{0}} & =\frac{1}{\int_{1}^{\infty} g_{\lambda}\left(y \mid H_{0}\right) d y} .
\end{aligned}
$$


In order to erase the low-reliability RS coded symbols, we assume that $\lambda_{T}$ is the threshold, which activates an erasure insertion, if $\lambda \leq \lambda_{T}$. Consequently, the correct symbol probability, $P_{c}$, and the random symbol error probability, $P_{t}$, after erasure insertion can be formulated as

$$
\begin{aligned}
P_{c} & =P\left(H_{1}\right) \cdot \int_{\lambda_{T}}^{\infty} f_{\lambda}\left(y \mid H_{1}\right) d y \\
& =P\left(H_{1}\right)-P\left(H_{1}\right) \cdot \int_{1}^{\lambda_{T}} f_{\lambda}\left(y \mid H_{1}\right) d y \\
P_{t} & =P\left(H_{0}\right) \cdot \int_{\lambda_{T}}^{\infty} f_{\lambda}\left(y \mid H_{0}\right) d y \\
& =P\left(H_{0}\right)-P\left(H_{0}\right) \cdot \int_{1}^{\lambda_{T}} f_{\lambda}\left(y \mid H_{0}\right) d y
\end{aligned}
$$

and the symbol erasure probability $P_{e}$ can be expressed as

$$
P_{e}=1-P_{c}-P_{t} .
$$

Again, if we assume that the symbol errors and symbol erasures within a codeword are independent due to the ideal interleaving, then the codeword decoding error probability after "errors-and-erasures" decoding can be expressed in the form of [15]

$$
\begin{aligned}
P_{W}= & \sum_{i=0}^{\mathcal{N}} \sum_{j=j_{0}(i)}^{\mathcal{N}-i}\left(\begin{array}{c}
\mathcal{N} \\
i
\end{array}\right)\left(\begin{array}{c}
\mathcal{N}-i \\
j
\end{array}\right) \\
& \cdot P_{t}^{i} P_{e}^{j}\left(1-P_{t}-P_{e}\right)^{\mathcal{N}-i-j}
\end{aligned}
$$

where $j_{0}(i)=\max \{0, \mathcal{N}-\mathcal{K}+1-2 i\}$, while $P_{t}$, and $P_{e}$ represent the random symbol error probability and symbol erasure probability, respectively, before decoding, which are given by (60) and (61).

Above, we have analyzed the performance of the uncoded and RS-coded DS-CDMA system using noncoherent $M$-ary orthogonal modulation, when diversity reception with EGC and SC were considered. Let us now evaluate the system's performance numerically.

\section{NUMERICAL RESULTS}

In this section the average bit or codeword decoding error probability of the DS-CDMA system using noncoherent $M$-ary orthogonal modulation - with or without RS coding - is evaluated as a function of the average signal-to-noise ratio (SNR) per bit $\bar{\gamma}_{b}$, that of the preset threshold $\lambda_{T}$, and (or) versus the number of simultaneous users in the system. The average SNR per bit is obtained by $\bar{\gamma}_{b}=L_{p} \bar{\gamma}_{c} / b$, where $b=\log _{2} M$.

Fig. 3 shows the BER performance of the EGC and SC schemes for $L=1,2,3,4,5$ diversity branches upon evaluating (36), (33), and (34) for EGC, and (37), (33), and (34) for SC. We assumed that an uncoded DS-CDMA system using 64-ary orthogonal modulation was employed, the receiver was capable of combining all the multipath signals, i.e., we had $L=L_{p}$, the number of simultaneous users was $K=10$, and the ratio of bit duration to chip duration was $N=128$. As expected, both the EGC and SC schemes provide BER

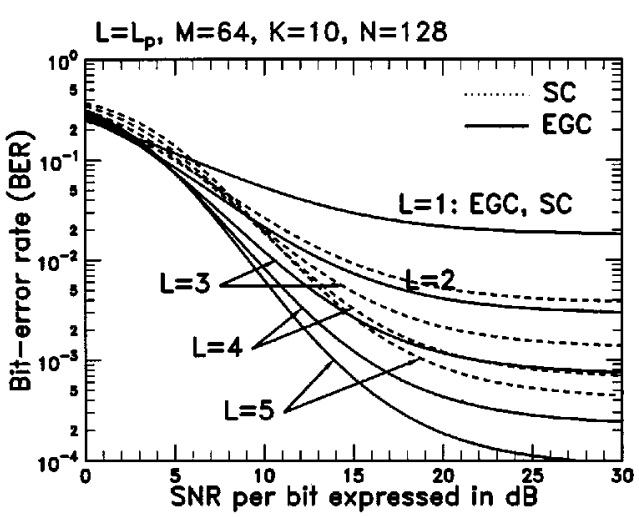

Fig. 3. EGC, SC: BER versus average SNR per bit for DS-CDMA systems using noncoherent 64-ary modulation without FEC, when the number of simultaneous users is $K=10$, the ratio of bit duration to chip duration is $N=128$, the receiver can combine all the multipath signals, i.e., $L=L_{p}$ and $L=1,2,3,4,5$. The results were evaluated from (36), (33), and (34) for EGC, while from (37), (33), and (34) for SC.

improvements for moderate to high SNRs per bit, when the number of combined diversity paths $L$ increases. Furthermore, the results show that the EGC scheme has a lower BER than the SC scheme for a given number of combined paths $L$ and for a given SNR per bit $\bar{\gamma}_{b}$. This is because EGC is the optimal diversity combining scheme for a noncoherent demodulation technique. However, BER floors are observed for both the EGC and SC schemes. This implies that in DS-CDMA systems, due to the multiple-access interference, the BER performance cannot be improved simply by increasing the transmission power alone. Multiuser detection [1] constitutes a powerful technique mitigating the multiple-access interference.

As an example, Fig. 4 shows the codeword decoding error probability of (62) over Rayleigh fading channels for both the EGC and SC schemes, when employing the RS $(32,20)$ code over the Galois field $\mathrm{GF}(32)=\mathrm{GF}\left(2^{5}\right)$ corresponding to 5-bit symbols using "errors-and-erasures" decoding. In these figures, the codeword decoding error probabilities were computed for different values of SNR per bit and for different thresholds, in order to find the optimum thresholds for the RTT-based erasure insertion scheme. From the results, we observe that for a constant SNR per bit $\bar{\gamma}_{b}$, there exists an optimum threshold, for which the "errors-and-erasures" decoding achieves the minimum codeword decoding error probability. Hence, an inappropriate threshold leads to higher codeword decoding error probability than the minimum seen in the figure. Observe furthermore that for both the EGC and SC schemes, the optimum threshold assumes values around 1.5-2.0, even though the SNR per bit changes over a large dynamic range from about 6 to $15 \mathrm{~dB}$.

Equivalently, in Fig. 5, the codeword decoding error probability of (62) was evaluated for different values of the threshold $\lambda_{T}$ and for different number of simultaneous users, in order to find the optimum thresholds for the RTT-based erasure insertion scheme versus the number of users and the SNR per bit. Similarly to our previous results in Fig. 4, we observe that for a constant number of simultaneous users $K$, there exists an optimum threshold, for which the "errors-and-erasures" decoding achieves the minimum codeword decoding error probability and an inappropriate threshold leads to higher codeword decoding 


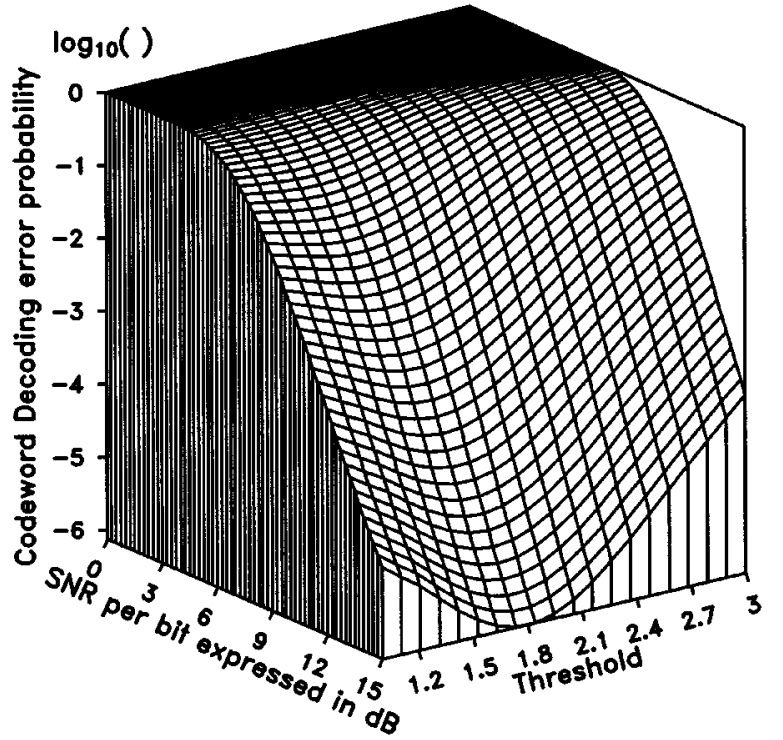

(a) EGC: $M=32, L=L_{p}=2, K=10, N=128, R S(32,20)$

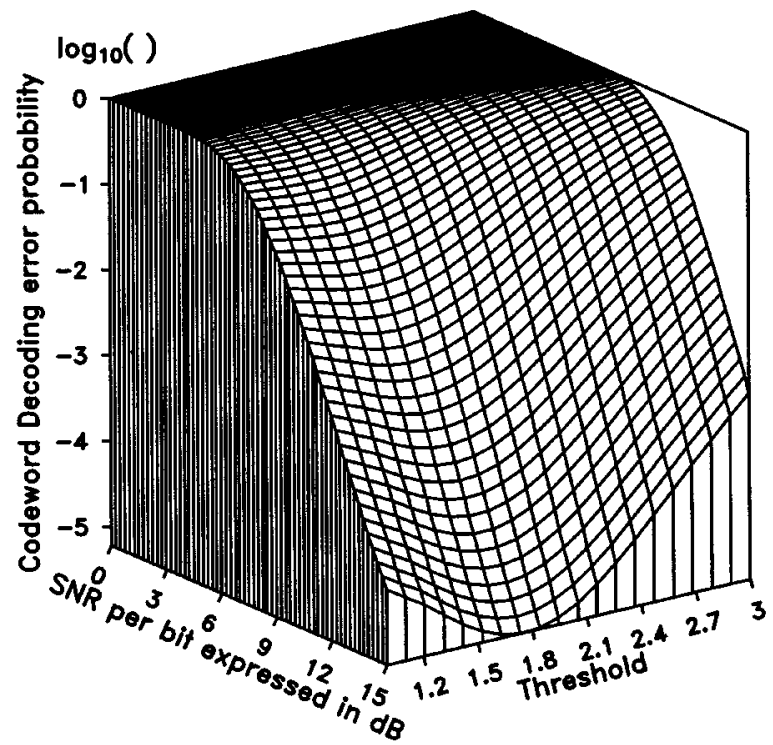

(b) SC: $M=32, L=L_{p}=2, K=10, N=128, R S(32,20)$

Fig. 4. Codeword decoding error probability versus the average SNR per bit, $\bar{\gamma}_{b}$ and the threshold, $\lambda_{T}$ for the RS(32,20) FEC code using "errors-and-erasures" decoding over Rayleigh fading channels, evaluated from (51)-(62).

error probability than the minimum seen in the figure. Moreover, we observe again that for both the EGC and SC schemes, the optimum threshold assumes values around 1.5-2.0, when the number of simultaneous users changes over a large dynamic range from about 20-100. However, due to the multiple-access interference, the codeword decoding error probability for any given value of threshold increases dramatically, when increasing the number of simultaneous users $K$.

In summary, from Figs. 4 and 5, we gain an explicit insight into the characteristics of Viterbi's RTT over the dispersive Rayleigh fading channels studied, suggesting that the optimum threshold was typically around 1.5-2. Since the optimum threshold can be derived numerically for a given number of

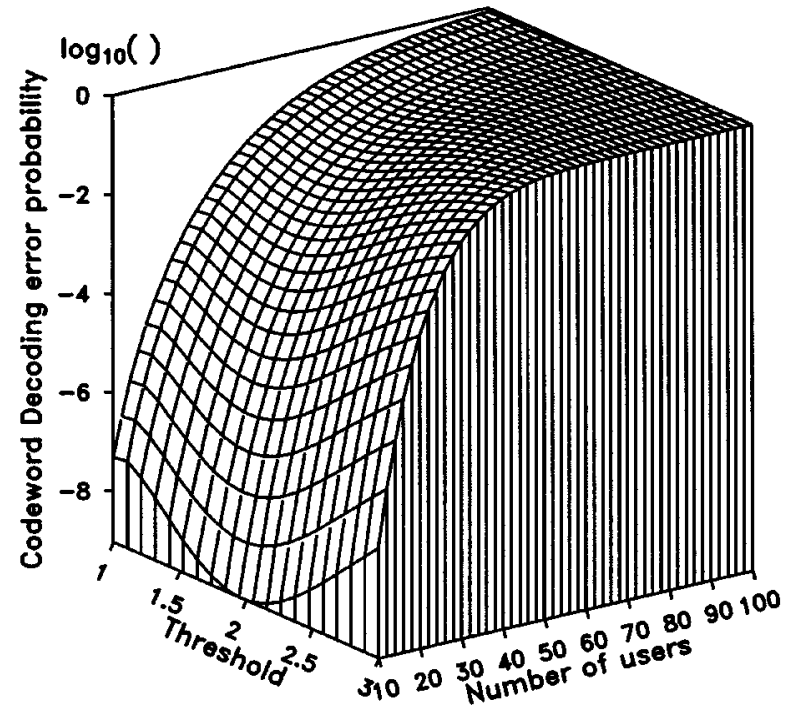

(a) EGC: $M=32, L=L_{p}=2, \bar{\gamma}_{b}=20 d B, N=128, R S(32,20)$

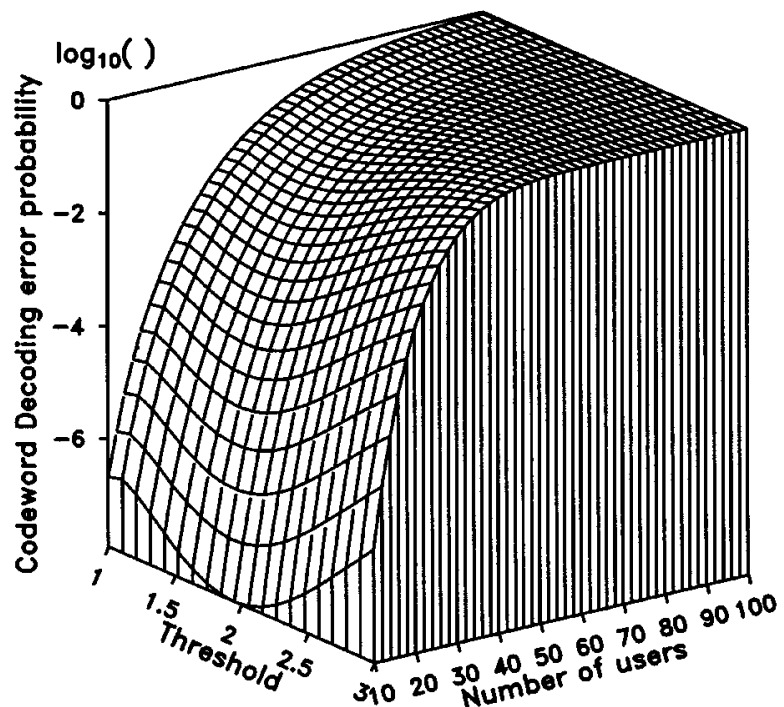

(b) SC: $M=32, L=L_{p}=2, \bar{\gamma}_{b}=20, N=128, R S(32,20)$

Fig. 5. Codeword decoding error probability versus the number of simultaneous users, $K$ and the threshold, $\lambda_{T}$ for the RS $(32,20)$ FEC code using "errors-and-erasures" decoding over Rayleigh fading channels. The results were evaluated from (51)-(62).

simultaneous users $K$, and for a given average SNR per bit $\bar{\gamma}_{b}$, in our further investigations we have assumed that the optimum threshold was employed, whenever "errors-and-erasures" decoding was used. However, the optimum threshold depends on the SNR per bit and slightly increases, when increasing the SNR per bit value.

In Fig. 6 we evaluated the codeword decoding error probability of an $M$-ary DS-CDMA system employing either "error-correction-only" or "errors-and-erasures" decoding. We assume that there were $L_{p}=5$ resolvable paths, and $L=1,2,3,4$, or 5 paths were actually combined in the receiver using an EGC [Fig. 6(a)] or SC [Fig. 6(b)] scheme. Again, we also assumed that an optimal threshold $\lambda_{T}$ was employed for any given value of the average received SNR per bit. The remaining parameters were specified in the figures. 


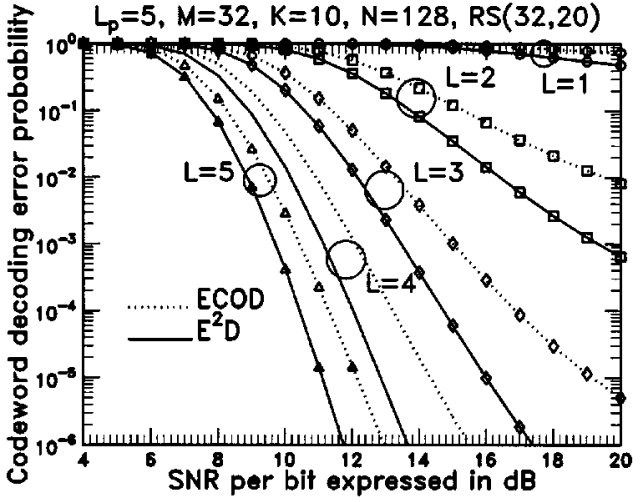

(a) EGC

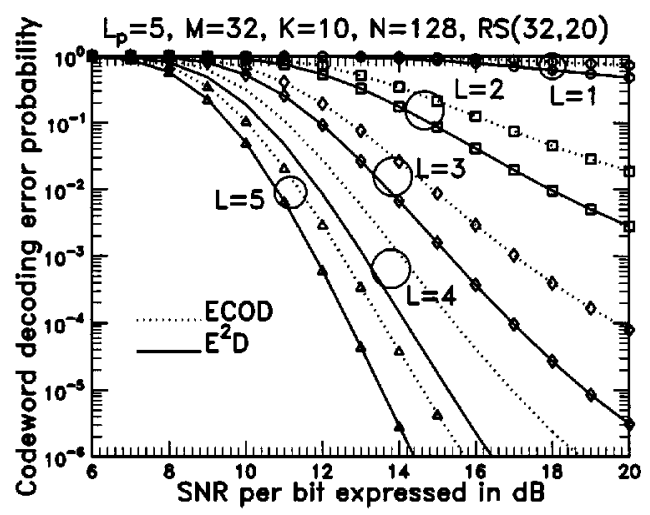

(b) $\mathrm{SC}$

Fig. 6. Codeword decoding error probability versus the average SNR per bit, $\bar{\gamma}_{b}$ for the RS(32,20) FEC code using "error-correction-only" decoding (ECOD) and "errors-and-erasures" decoding $\left(E^{2} \mathrm{D}\right)$ with parameters of $M=\mathcal{N}=32$, $\mathcal{K}=20, K=10, N=128, L_{p}=5$ resolvable multipath components and a receiver diversity combining capability of $L=1,2,3,4,5$ over dispersive Rayleigh fading channels.

The results show that under dispersive multipath Rayleigh fading conditions, while assuming a constant average SNR per bit and a constant number of diversity components combined, the decoding algorithms using "errors-and-erasures" decoding based on the RTT erasure insertion scheme outperform "error-correction-only" decoding. As shown in Fig. 6(a) for EGC, for $L=3,4,5$ and at the error probability of $10^{-3}$, the "errors-and-erasures" decoding scheme can achieve a gain of about $1.6,1$, and $0.8 \mathrm{~dB}$, respectively, over the "error-correction-only" decoding scheme. Similarly, for $L=4,5$ and at the error probability of $10^{-6}$, the "errors-and-erasures" decoding scheme can achieve a gain of about 1.8 and $1.2 \mathrm{~dB}$, respectively, over the "error-correction-only" decoding scheme. Similar results can also be observed in Fig. 6(b) for SC.

Similarly, Fig. 7 shows the codeword decoding error probability of the $\operatorname{RS}(32,20)$ code as a function of the number of simultaneous users, $K$, for parameters of $\bar{\gamma}_{b}=20 \mathrm{~dB}$, $N=128, L=L_{p}=1,2,3$ over dispersive multipath Rayleigh fading channels. The codeword decoding error probability of the "error-correction-only" and "errors-and-erasures" decoding scheme was compared for the EGC and SC considered. We assumed that the receiver used the optimum threshold for any number of users. The results show that for a constant number of users, for a constant number of diversity components

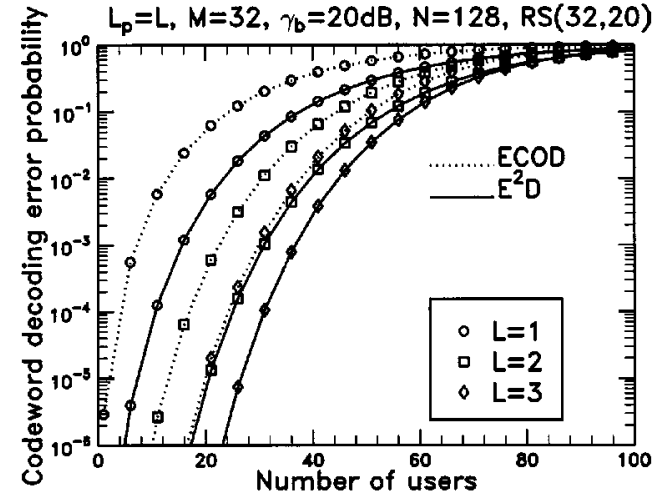

(a) EGC

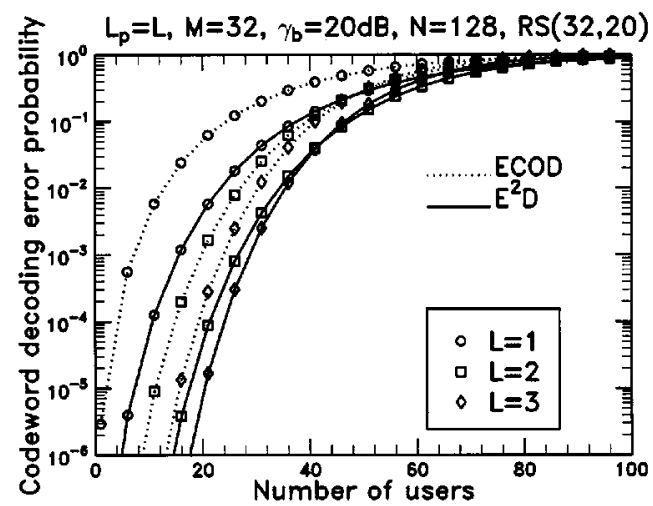

(b) $\mathbf{S C}$

Fig. 7. Codeword decoding error probability versus the number of simultaneous users, $K$ for the RS(32,20) FEC code using "error-correction-only" decoding (ECOD) and "errors-and-erasures" decoding $\left(E^{2} \mathrm{D}\right)$ with parameters of $M=\mathcal{N}=32, \mathcal{K}=20, \bar{\gamma}_{b}=20 \mathrm{~dB}, N=128$, $L=L_{p}=1,2,3$ over dispersive Rayleigh fading channels.

combined, and upon invoking the optimum threshold, the codeword decoding error probability of the system employing "errors-and-erasures" decoding based on the RTT erasure insertion scheme was lower than that employing "error-correction-only" decoding. At the codeword decoding error probability of $10^{-6}$, the EGC-assisted "errors-and-erasures" scheme in Fig. 7(a) can support 7 or 6 more simultaneous users for $L=2$ or 3, respectively, than the "error-correction-only" scheme. By contrast, the SC-aided "errors-and-erasures" scheme in Fig. 7(b) can support 6 or 4 more simultaneous users for $L=2$ or 3, respectively, than the "error-correction-only" scheme.

Finally, in Fig. 8 we evaluated the influence of the code rate, $R_{c}$, on the codeword decoding error probability of the DS-CDMA systems using both "error-correction-only" decoding and "errors-and-erasures" decoding, for both the EGC and the SC combining schemes investigated. The codeword decoding error probability was evaluated as a function of the number of simultaneous users for the code rates of $R_{c}=7 / 8,3 / 4,5 / 8$, and $1 / 2$. We assumed that the 64 symbol long RS code family of $\mathrm{RS}(64, \mathcal{K})$ over the Galois field $\mathrm{GF}(64)=\mathrm{GF}\left(2^{6}\right)$ using "error-correction-only" and "errors-and-erasures" employing RTT was invoked. As expected, the results imply that for all of the code rates considered, the 


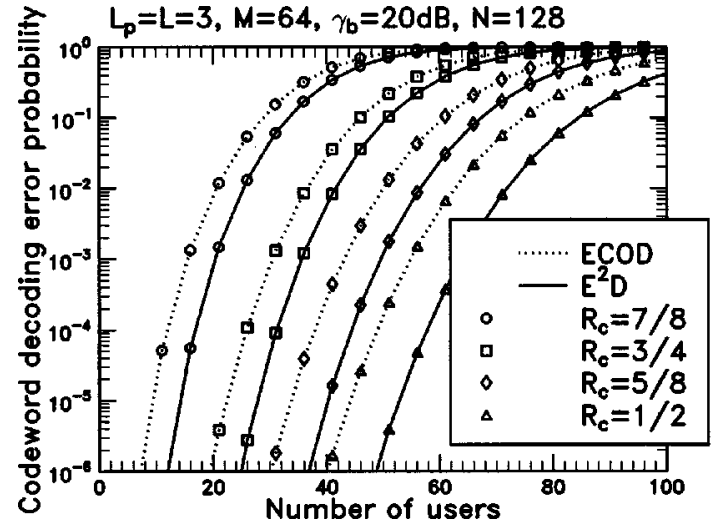

(a) EGC

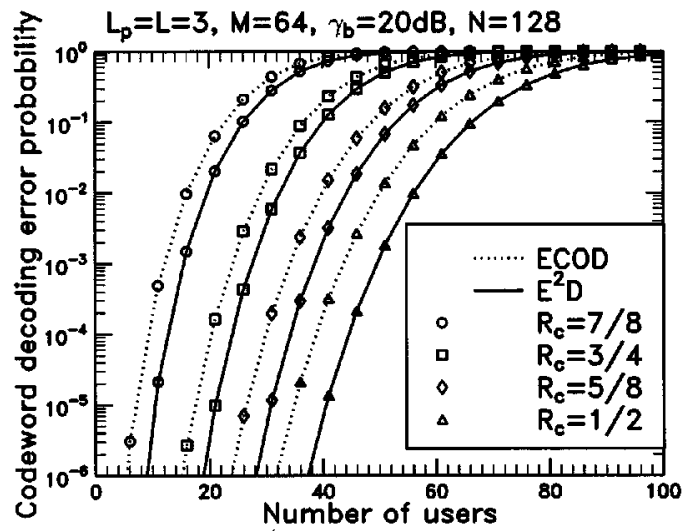

(b) SC

Fig. 8. Codeword decoding error probability versus the number of simultaneous users, $K$ for various RS FEC codes using "error-correction-only" decoding (ECOD) and "errors-and-erasures" decoding $\left(E^{2} \mathrm{D}\right)$ at different code rates with parameters of $M=\mathcal{N}=64, \mathcal{K}=32,40,48$, and 56 , i.e., $R_{c}=1 / 2,5 / 8,3 / 4$ and $7 / 8, \bar{\gamma}_{b}=20 \mathrm{~dB}, N=128, L=L_{p}=3$ over dispersive Rayleigh fading channels.

decoding scheme using "errors-and-erasures" employing RTT outperformed the arrangement using "error-correction-only" decoding. For a constant codeword decoding error probability and for a given code rate, the DS-CDMA systems employing RS codes using "errors-and-erasures" decoding can support 4 to 8 more simultaneous users at the codeword decoding error probability of $10^{-6}$ than those employing "error-correction-only" decoding.

\section{CONCLUSION}

In summary, in this paper the performance of RS-coded DS-CDMA systems using "errors-and-erasures" decoding has been investigated and compared to that using "error-correction-only" decoding over dispersive multipath Rayleigh fading channels, when noncoherent $M$-ary orthogonal modulation and diversity reception using EGC or SC schemes were considered. Viterbi's RTT technique has been studied, in order to quantify the reliability of the received RS coded symbols. The symbols having a low-confidence were erased before RS "errors-and-erasures" decoding. The PDFs associated with the RTT under the hypotheses of correct detection and erroneous detection of the $M$-ary signals have been derived and the op- timum thresholds have been determined. Our numerical results showed that by using "errors-and-erasures" decoding associated with the RTT, RS codes of a given code rate can achieve a higher coding gain than upon using "error-correction-only" decoding without erasure information. DS-CDMA systems using the proposed "errors-and-erasures" decoding could also support more simultaneous users than without erasure information. Moreover, the numerical results showed that the optimum threshold for the RTT over multipath Rayleigh fading channels was around $[1.5,2]$ for the practical range of average SNR per bit values and for a given number of simultaneous users.

\section{APPENDIX}

THE DERIVATION OF (37)

In this Appendix we derive the correct symbol probability, $P\left(H_{1}\right)$, of the SC scheme. $P\left(H_{1}\right)$ associated with the SC scheme can be expressed in the form of (35) with $f_{U_{m}^{i}}(x)$ and $f_{U_{j}^{i}}(y)$ given by (28) and (31), respectively. In order to derive a general result, we let $\lambda_{1}=1 /\left(1+\bar{\gamma}_{c}\right)$ and $\lambda_{2}=1$. Then, (28) and (31) can be expressed as

$$
\begin{aligned}
f_{U_{m}^{i}}(y) & =\lambda_{2} L \exp \left(-\lambda_{2} y\right)\left[1-\exp \left(-\lambda_{2} y\right)\right]^{L-1} \\
f_{U_{j}^{i}}(y) & =\lambda_{1} L \exp \left(-\lambda_{1} y\right)\left[1-\exp \left(-\lambda_{1} y\right)\right]^{L-1} .
\end{aligned}
$$

With the aid of (63), it can be shown that

$$
\int_{0}^{y} f_{U_{m}^{i}}(x) d x=\left[1-\exp \left(-\lambda_{2} y\right)\right]^{L} .
$$

Substituting (64) and (65) into (35), $P\left(H_{1}\right)$ of the SC scheme can be expressed as

$$
\begin{aligned}
P\left(H_{1}\right)= & \int_{0}^{\infty} \lambda_{1} L \exp \left(-\lambda_{1} y\right)\left[1-\exp \left(-\lambda_{1} y\right)\right]^{L-1} \\
& \cdot\left[1-\exp \left(-\lambda_{2} y\right)\right]^{L(M-1)} d y \\
= & \sum_{k=0}^{L-1}(-1)^{k}\left(\begin{array}{c}
L-1 \\
k
\end{array}\right) \lambda_{1} L \int_{0}^{\infty} \exp \left[-(1+k) \lambda_{1} y\right] \\
& \cdot\left[1-\exp \left(-\lambda_{2} y\right)\right]^{L(M-1)} d y
\end{aligned}
$$

Upon using the relationship of

$$
\begin{aligned}
\int_{0}^{\infty} & \exp \left[-\left((1+k) \lambda_{1}+n \lambda_{2}\right) y\right]\left[1-\exp \left(-\lambda_{2} y\right)\right]^{L(M-1)-n} d y \\
= & \frac{[L(M-1)-n] \lambda_{2}}{(1+k) \lambda_{1}+n \lambda_{2}} \int_{0}^{\infty} \\
& \cdot \exp \left[-\left((1+k) \lambda_{1}+(n+1) \lambda_{2}\right) y\right] \\
& \cdot\left[1-\exp \left(-\lambda_{2} y\right)\right]^{L(M-1)-n-1} d y
\end{aligned}
$$

the integral in (66) can be expressed as

$$
\begin{gathered}
\int_{0}^{\infty} \exp \left[-(1+k) \lambda_{1} y\right]\left[1-\exp \left(-\lambda_{2} y\right)\right]^{L(M-1)} d y \\
=\frac{1}{(1+k) \lambda_{1}} \prod_{n=1}^{L(M-1)} \frac{n}{n+(1+k) \lambda_{1} / \lambda_{2}}
\end{gathered}
$$

Substituting the above equation into (66) and letting $m=1+k$, it can be shown that $P\left(H_{1}\right)$ can be expressed as

$$
P\left(H_{1}\right)=\sum_{m=1}^{L}(-1)^{m+1}\left(\begin{array}{c}
L \\
m
\end{array}\right) \prod_{n=1}^{L(M-1)} \frac{n}{n+m \lambda_{1} / \lambda_{2}} .
$$


Finally, replacing $\lambda_{1}$ by $1 /\left(1+\bar{\gamma}_{c}\right)$ and $\lambda_{2}$ by 1 in (69), the closed form of the correct symbol probability, $P\left(H_{1}\right)$ associated with the SC scheme can be obtained as shown in (37).

\section{ACKNOWLEDGMENT}

The authors thank the anonymous reviewers for their constructive critique.

\section{REFERENCES}

[1] S. Verdu, Multiuser Detection. Cambridge, U.K.: Cambridge University Press, 1998.

[2] K. S. Gilhousen, et al., "On the capacity of a cellular CDMA system," IEEE Trans. Veh. Technol., vol. 40, pp. 303-312, May 1991.

[3] EIA/TIA-95 Rev A, "Mobile station-base station compatibility standard for dual-mode wideband spread spectrum cellular system,", 1995.

[4] P. K. Enge and D. V. Sarwate, "Spread-spectrum multiple-access performance of orthogonal codes: Linear receivers," IEEE Trans. Commun., vol. COM-35, pp. 1309-1319, Dec. 1987.

[5] — , "Spread-spectrum multiple-access performance of orthogonal codes: Impulsive noise," IEEE Trans. Commun., vol. COM-36, pp. 98-105, Jan. 1988.

[6] M. Chase and K. Pahlavan, "Performance of DS-CDMA over measured indoor radio channels using random orthogonal codes," IEEE Trans. Veh. Technol., vol. 42, pp. 617-624, Nov. 1993.

[7] L.-L. Yang and C.-S. Li, "DS-CDMA performance of random orthogonal codes over Nakagami multipath fading channels," in Proc. IEEE ISSSTA '96, Mainz, Germany, Sept. 22-25, 1996, pp. 1282-1286.

[8] L. M. A. Jalloul and J. M. Holtzman, "Performance analysis of DS/CDMA with noncoherent $M$-ary orthogonal modulation in multipath fading channels," IEEE J. Select. Areas Commum., vol. 12, pp. 862-870, Sept. 1994

[9] V. Aalo, O. Ugweje, and R. Sudhakar, "Performance analysis of a DS/CDMA system with noncoherent $M$-ary orthogonal modulation in Nakagami fading," IEEE Trans. Veh. Technol., vol. 47, pp. 20-29, Feb. 1998.

[10] S. W. Kim and W. Stark, "Performance limits of Reed-Solomon coded CDMA with orthogonal signaling in a Rayleigh-fading channel," IEEE Trans. Commun., vol. 46, pp. 1125-1134, Sept. 1998.

[11] S. Lin and D. J. Costello, Error Control Coding: Fundamentals and Applications. Englewood Cliffs, NJ: Prentice-Hall, 1983.

[12] G. D. Forney, "Exponential error bounds for erasure, list, and decision feedback scheme," IEEE Trans. Inform. Theory, vol. IT-14, pp. 206-220, Mar. 1968.

[13] A. J. Viterbi, "A robust ratio-threshold technique to mitigate tone and partial band jamming in coded MFSK systems," in IEEE Military Commun. Conf. Rec., Oct. 1982, pp. 22.4.1-22.4.5.

[14] L. F. Chang and R. J. McEliece, "A study of Viterbi's ratio threshold AJ technique," in IEEE Military Commun. Conf. Rec., Oct. 1984, pp. 11.2.1-11.2.5.

[15] C. W. Baum and M. B. Pursley, "Bayesian methods for erasure insertion in frequency-hop communication system with partial-band interference," IEEE Trans. Commun., vol. 40, pp. 1231-1238, July 1992.

[16] _ "A decision-theoretic approach to the generation of side information in frequency-hop multiple-access communications," IEEE Trans. Commun., vol. 43, pp. 1768-1777, Feb./Mar./Apr. 1995.

[17] — "Bayesian generation of dependent erasures for frequency-hop communications and fading channels," IEEE Trans. Commun., vol. 44, pp. 1720-1729, Dec. 1996

[18] , "Erasure insertion in frequency-hop communications with fading and partial-band interference," IEEE Trans. Veh. Technol., vol. 46, pp. 949-956, Nov. 1997.

[19] L.-L. Yang and L. Hanzo, "Performance analysis of coded $M$-ary orthogonal signaling using errors-and-erasures decoding over frequencyselective fading channels," IEEE J. Select. Areas Commun., to be published.

[20] C. M. Keller and M. B. Pursley, "Diversity combining for channels with fading and partial-band interference," IEEE J. Select. Areas Commun. vol. SAC-5, pp. 248-260, Feb. 1987.
[21] T. Eng, N. Kong, and L. B. Milstein, "Comparison of Diversity combining techniques for Rayleigh-fading channels," IEEE Trans. Commun., vol. 44, pp. 1117-1128, Sept. 1996

[22] J. G. Proakis, Digital Communications, 3rd ed. New York: McGrawHill, 1995.

[23] G.-T. Chyi, J. G. Proakis, and C. M. Keller, "On the symbol error probability of maximum-selection diversity reception schemes over a Rayleigh fading channel," IEEE Trans. Commun., vol. 37, pp. 79-83, Jan. 1989.

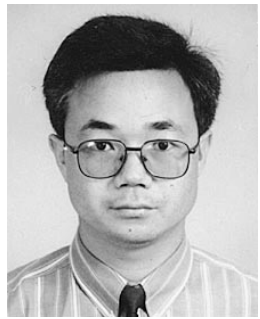

Lie-Liang Yang (M'98) received the B.Eng. degree in communication engineering from Shanghai TieDao University in 1988, and the M.S., Ph.D degrees in communications and electronics from Northern Jiaotong University in 1991 and 1997 respectively.

From 1991-1993 he was a Lecturer with the Department of Electrical Engineering, East-China Jiaotong University, China. From 1993-1997 he was with the Modern Communications Research Institute, Northern Jiaotong University, China. From June-December 1997, he was a Visiting Scientist of the Institute of Radio Engineering and Electronics, Academy of Sciences of the Czech Republic. Since December 1997, he has been with the Communication Group, Department of Electronics and Computer Science, University of Southampton, U.K. and is involved in researching various error correction coding, modulation, synchronization and spread spectrum systems for future generations of wireless mobile communication systems. He has published over 50 papers in journals and conference proceedings and he was awarded the Royal Society Sino-British Fellowship in 1997.

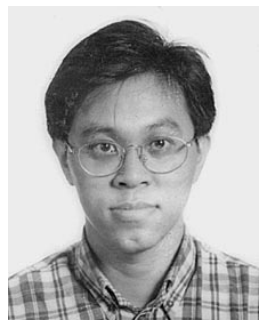

Kai Yen (S'00) was born in Singapore in 1969. He received the B.Eng. degree with honors in electrical and electronics engineering from the Nanyang Technological University, Singapore in 1996. From 1996-1997, he was with the Centre for Wireless Communications (CWC) at the National University of Singapore as a Research Engineer. Currently he is working toward the Ph.D. degree at the University of Southampton, U.K. funded by a scholarship from CWC. His research interests include multiuser detection and modem techniques for CDMA.

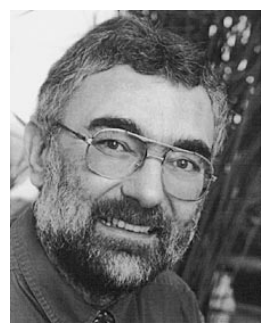

Lajos Hanzo (M'91-SM'92) graduated in electronics in 1976 and in 1983 he was conferred a Ph.D. During his 24-year career in telecommunications he has held various research and academic posts in Hungary, Germany and the U.K. Since 1986 he has been with the Department of Electronics and Computer Science, University of Southampton, U.K. and has been a Consultant to Multiple Access Communications Ltd., U.K. Currently he holds the Chair in Telecommunications. He coauthored five books on mobile radio communications, published over 300 research papers, organized and chaired conference sessions, presented overview lectures and was awarded a number of distinctions. Currently he is managing a research team, working on a range of research projects in the field of wireless multimedia communications under the auspices of the Engineering and Physical Sciences Research Council (EPSRC) U.K., the European IST Programme and the Mobile Virtual Centre of Excellence (VCE). He also provides a range industrial training courses. For further information on research in progress and associated publications please refer to the www home-page http://www-mobile.ecs.soton.ac.uk 